\title{
Interfacial spin-flip-generated charge pumping
}

\author{
Sang-Chan Lee $\odot,{ }^{*}$ Suik Cheon $\odot,{ }^{\dagger}$ and Hyun-Woo Lee $\odot^{\ddagger}$ \\ Department of Physics, Pohang University of Science and Technology, Pohang 37673, Korea
}

(Received 1 December 2020; accepted 9 February 2021; published 23 February 2021)

\begin{abstract}
Precessing magnetization is known to generate and inject pure spin currents to adjacent materials by the spin pumping effect. However, the generation of the pure spin current is the only case of the spin pumping at the spin-conserving interface. The charge current is usually accompanied by the usual spin pumping effect at the spin-nonconserving interface in general. In this paper, we consider systems where spin can be scattered and flipped by the Elliott-Yafet-like process at interfaces. For these systems, we investigate the interface-generated charge pumping by using the adiabatic quantum pumping theory. We present the analytic expressions for this pumped charge current in terms of the generalized mixing conductances. For the system that we consider, we find interestingly that the connection between the spin pumping effects and magnetoelectronic circuit theory, which has been demonstrated for the spin-conserving systems, can be extended in spin-nonconserving interfaces, and we show that the pumped charge current generated from the interfacial spin-flip potential can be understood in the context of the generalized spin conductance tensors suggested from the generalized magnetoelectronic circuit theory. We also compare the pumped charge current with other mechanisms that generate a charge current or electric voltage in the presence of the precessing magnetization.
\end{abstract}

DOI: 10.1103/PhysRevResearch.3.013177

\section{INTRODUCTION}

The pioneering theories of the spin pumping [1-3] and the magnetoelectronic circuit [4-6] have provided important theoretical tools to describe various spin transport phenomena through interfaces such as spin-transfer torque [7-10], spin-orbit torque [11,12], spin memory loss [13-15], and, of course, the spin pumping effect itself [1-3], which has been widely used for a decade as a standard method to generate a pure spin current in various experiments.

The original versions of the spin pumping theory [1] and the magnetoelectronic circuit theory [4] have a clear limitation, however, since the theories assume the longitudinal spin component along the magnetization direction to be conserved, whereas this assumption may be violated in experiments. In particular, this assumption may be seriously violated in systems with strong spin-orbit coupling, which is an essential part of the spintronics in recent years [16-18]. So various attempts to generalize the original theories to spin-nonconserving systems have been made [13-15,19-33]. The spin pumping current in the presence of the Rashba spin-orbit coupling and the origin of spin-memory loss at the interface with heavy

\footnotetext{
*darau@ postech.ac.kr

†enprodigy@postech.ac.kr

"hwl@ postech.ac.kr
}

Published by the American Physical Society under the terms of the Creative Commons Attribution 4.0 International license. Further distribution of this work must maintain attribution to the author(s) and the published article's title, journal citation, and DOI. metals have been studied and have demonstrated that the Rashba spin-orbit coupling can give rise to the spin-memory loss [21]. In this way, the spin pumping effects and its spin pumping currents are widely investigated and generalized in the presence of the spin-orbit interaction at the interface and bulk of the materials.

Here, we study the charge pumping by precessing magnetization in spin-nonconserving systems. In a spin-conserving system, where the magnetoelectric coupling is absent, the charge pumping is prohibited [1] and a precessing uniform magnetization pumps a pure spin current to surrounding materials. In a spin-nonconserving system, on the other hand, the magnetoelectric coupling is possible. It has been indeed argued [24] based on the Onsager's reciprocity that the charge pumping may be possible in certain types of spinnonconserving systems. Then the charge pumping is allowed and thus the pumped spin current may be accompanied by the pumped charge current in principle. The charge pumping may induce a voltage difference between ferromagnets with precessing magnetization and their adjacent materials, and this voltage may induce additional spin current between the ferromagnets and adjacent materials. Thus the charge pumping can affect the magnitude of the spin current through interfaces and make experimental analysis of spin pumping complicated. Nevertheless the charge pumping has not received due attention. In this work, we consider the Elliott-Yafet-like type of the spin-nonconserving potential at the interface between two metallic layers and theoretically examine effects of the interfacial spin-flip potential on the charge pumping using the adiabatic quantum pumping theory [34-36]. We show that this interfacial spin-flip-generated charge pumping does not vanish in general. In addition, we demonstrate that the pumped charge current can be expressed in terms of the 
generalized mixing conductance tensor proposed recently by the generalized magnetoelectronic circuit theory $[15,20]$.

The rest of the paper is organized as follows. In Sec. II, we briefly summarize the adiabatic quantum pumping theory and apply this theory to systems with the spin-nonconserving interfaces. We use a model Hamiltonian with the interfacial spin-flip potential and calculate the scattering matrix explicitly from the model Hamiltonian. Then we derive the expression for the pumped charge current in terms of the scattering matrix and show that the pumped charge current does not generally vanish in the presence of the interfacial spin-flip potential. In Sec. III, we investigate the connection between the adiabatic pumping theory and the magnetoelectronic theory in systems with spin-nonconserving potential at interfaces, and we show that the pumped charge current derived from the adiabatic pumping theory can be directly connected to the generalized conductance tensors defined in the generalized magnetoelectronic circuit theory. We also compare the other mechanisms that can generate a charge current or an electric voltage from the precession of the magnetization. Section IV summarizes our paper.

\section{FORMALISM AND RESULT}

\section{A. Adiabatic quantum pumping}

The adiabatic quantum pumping refers to a phenomenon in which an adiabatic variation of system parameters can generate electron flow in the absence of any direct chemical potential biases or temperature gradients [34-36]. The flow may depend on electron's spins and thus the flow may generate in principle both charge current and spin current. When the variation is periodic, the dc component of the flow can be finite and its magnitude is proportional to the geometric area of the contour that the time-dependent variation of system parameters has made [34]. In systems that contain ferromagnetic materials, where the majority and minority electrons are subject to different environments, the pumped currents from the majority and minority electrons may be different, resulting in a spin current. This is the so-called spin pumping effect. In magnetic systems where the longitudinal spin component along the magnetization is conserved, the pumped charge currents from the majority and minority electrons cancel each other and only a spin current is pumped [1-3].

To investigate the charge pumping and the spin pumping simultaneously, it is useful to introduce a $2 \times 2$ current tensor, $\hat{I}^{\text {pump }}$, from which the pumped charge current $I_{\text {charge }}^{\text {pump }}$ and the pumped spin current $\boldsymbol{I}_{\text {spin }}^{\text {pump }}$ can be obtained as follows:

$$
\begin{aligned}
I_{\text {charge }}^{\text {pump }} & =\operatorname{Tr}\left[\hat{\sigma}_{0} \hat{I}^{\text {pump }}\right], \\
\boldsymbol{I}_{\text {spin }}^{\text {pump }} & =\frac{\hbar / 2}{-e} \operatorname{Tr}\left[\hat{\boldsymbol{\sigma}} \hat{I}^{\text {pump }}\right] .
\end{aligned}
$$

Here $\hat{\sigma}$ is the conventional spin Pauli matrix, and $\hat{\sigma}_{0}$ denotes the $2 \times 2$ identity matrix in the spin space. In a multiterminal system, the adiabatically pumped current tensor $\left(\hat{I}_{l}^{\text {pump }}\right)$ pumped into the terminal $l$ that resulted from the infinitesimal change $\delta X$ of the system parameter $X$ reads as follows:

$$
\begin{aligned}
\hat{I}_{l}^{\text {pump }} & =e \frac{\partial \hat{n}_{l}}{\partial X} \frac{d X(t)}{d t} \\
\frac{\partial \hat{n}_{l}}{\partial X} & =\left(\frac{1}{4 \pi i} \sum_{m n l^{\prime}} \frac{\partial \hat{s}_{m n, l l^{\prime}}}{\partial X} \hat{s}_{m n, l l^{\prime}}^{\dagger}\right)+\text { H.c., }
\end{aligned}
$$

where $\partial \hat{n}_{l} / \partial X$ amounts to the emissivity tensor that describes how many charges/spins are pumped into the terminal $l$ by the infinitesimal change $\delta X$ in the parameter space, and $\hat{s}_{m n, l l^{\prime}}$ is the conventional $2 \times 2$ scattering matrix in spin space. Here, $m$ and $n$ denote the transport channels, and $l$ and $l^{\prime}\left(l\right.$ and $l^{\prime}$ can be either 1 or 2 in the case of the two-terminal system) label the terminals to which the pumped current flows out. The precession of the magnetization can be considered as a two-dimensional parametric pump in spin space, so $\hat{I}_{l}^{\text {pump }}$ for the magnetization precession may contain dc components.

In the rest of the paper, we consider only a two-terminal system. For the terminal, say $l=2$, Eq. (2) may be written as follows:

$$
\hat{I}_{2}^{\text {pump }}=\frac{e}{4 \pi i} \sum_{m n}\left(\frac{\partial \hat{r}_{m n}}{\partial X} \hat{r}_{m n}^{\dagger}+\frac{\partial{\hat{t^{\prime}}}_{m n}}{\partial X}{\hat{t^{\prime}}}_{m n}^{\dagger}\right) \frac{d X}{d t}+\text { H.c. },
$$

where $\hat{r}_{m n}$ and $\hat{t}_{m n}^{\prime}$ correspond to $\hat{s}_{m n, 22}$ and $\hat{s}_{m n, 21}$ in Eq. (2), respectively. Thus $\hat{r}_{m n}$ is the reflection matrix for electrons reflected from channel $n$ in terminal 2 into channel $m$ in terminal 2 , and $\hat{t}_{m n}^{\prime}$ is the transmission matrix for electrons transmitted from channel $n$ in terminal 1 into channel $m$ in terminal 2.

When the spin component along the magnetization direction is conserved, $\hat{r}_{m n}$ and $\hat{t}_{m n}^{\prime}$ become diagonal in the spin space if the magnetization direction is used as the spin quantization axis. In this case, the pumped current tensor $\tilde{I}_{2}^{\text {pump }}$ becomes traceless [1-3] and the pumped charge current $\left(I_{2, \text { charge }}^{\text {pump }}\right)$ vanishes [Eq. (1a)]. On the other hand, the spin pumping current $\left(I_{2, \text { spin }}^{\text {pump }}\right)$ is finite and reads

$$
\boldsymbol{I}_{2, \text { spin }}^{\text {pump }}=\frac{\hbar}{4 \pi}\left[\operatorname{Re}\left(g^{\uparrow \downarrow}\right) \boldsymbol{m} \times \dot{\boldsymbol{m}}-\operatorname{Im}\left(g^{\uparrow \downarrow}\right) \dot{\boldsymbol{m}}\right],
$$

where the interface parameters $\operatorname{Re}\left(g^{\uparrow \downarrow}\right)$ and $\operatorname{Im}\left(g^{\uparrow \downarrow}\right)$ are the real part and the imaginary part of the spin-mixing conductance, respectively [2]. Therefore the precessing ferromagnet with a spin-conserving interface can be regarded as a pure spin current generator [2].

\section{B. Pumping in the presence of the interfacial spin-flip potential}

When the interface does not conserve the spin angular momentum during the scattering, $\hat{r}_{m n}$ and $\hat{t}_{m n}^{\prime}$ can acquire off-diagonal elements, which can modify various properties of the pumped current. For example, when the incident spin direction is collinear to the magnetization direction, and if the magnetization direction is used as the spin quantization axis, the reflection matrix reads

$$
\hat{r}_{m n}^{c}=\left(\begin{array}{cc}
r_{m n}^{\uparrow} & r_{m n}^{+} \\
r_{m n}^{-} & r_{m n}^{\downarrow}
\end{array}\right),
$$

where $r_{m n}^{\uparrow(\downarrow)}$ are the conventional spin-dependent reflection coefficients for up (down) spin components, and $r_{m n}^{+(-)}$ 


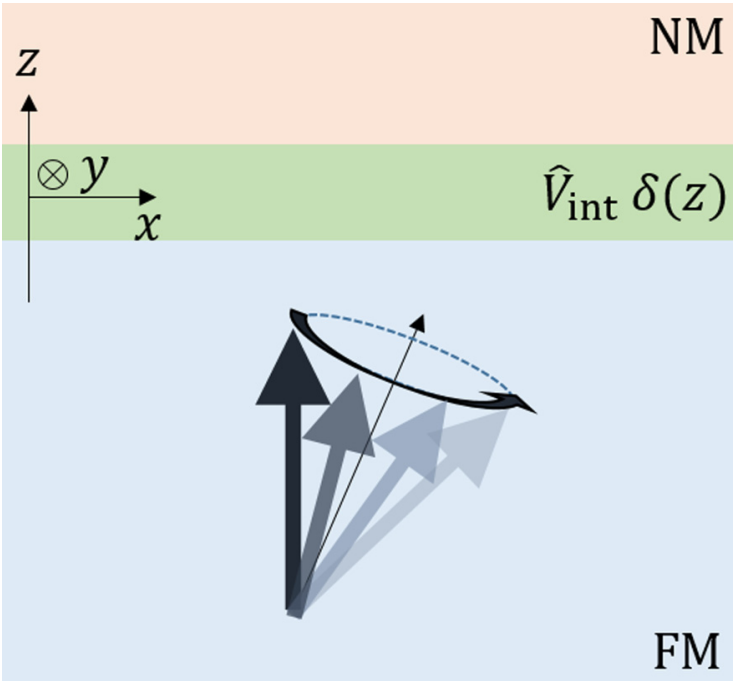

FIG. 1. Schematic diagram of a ferromagnetic metal (blue area) |nonmagnetic metal (red area) bilayer system $(\mathrm{F} \mid \mathrm{N})$ with the interfacial spin-flip potential $\left(\hat{V}_{\text {int }}\right.$, green area). The interfacial spinflip potential is confined to the interface and thus is proportional to $\delta(z)$.

correspond to the down-to-up (up-to-down) spin-flip scattering coefficients. For a spin-conserving interface, $r_{m n}^{+(-)}$ vanishes. To investigate the effects of the off-diagonal elements, we explicitly calculate the scattering matrix for the specific pumping model systems with the interfacial spin-flip potential.

\section{1. $F \delta \mid N$ bilayer system with interfacial spin-flip potential}

First, we introduce a $\mathrm{F} \delta \mid \mathrm{N}$ bilayer system (Fig. 1) that consists of a ferromagnetic metallic layer on the lower side $(z<0)$ and a nonmagnetic metallic layer on the upper side $(z>0)$. Here the symbol $\delta$ at the $\mathrm{F} \mid \mathrm{N}$ interface $(z=0)$ denotes that this interface hosts the interfacial spin-flip potential. It is assumed that a spin-flip potential exists at the interface $(z=0)$. To model this bilayer system, we take the following simple Hamiltonian,

$$
\hat{H}=\frac{\hat{p}^{2}}{2 m}+J_{\mathrm{ex}} \Theta(-z) \hat{\boldsymbol{\sigma}} \cdot \boldsymbol{m}+\hat{V}_{\mathrm{int}} \delta(z),
$$

where $\Theta(z)$ is the Heaviside theta function, $\delta(z)$ is the Dirac delta function, and $\boldsymbol{m}$ is a unit vector along the magnetization direction of the ferromagnetic layer. Here $\hat{V}_{\text {int }}$ is a $2 \times 2$ Hermitian matrix in spin space that may induce the spin flip. Its main effect is to modify the boundary conditions at the interface. For eigenstates with the in-plane momentum $\boldsymbol{k}_{\|}$and the energy $E$, the boundary conditions for the eigenstate wave functions $\psi_{\boldsymbol{k}_{\|}, E}^{\mathrm{F}}(\boldsymbol{r})=\exp \left(i \boldsymbol{k}_{\|} \cdot \boldsymbol{r}\right) \varphi_{\boldsymbol{k}_{\|}, E}^{\mathrm{F}}(z)$ in the ferromagnetic layer $(z<0)$ and $\psi_{\boldsymbol{k}_{\|}, E}^{\mathrm{N}}(\boldsymbol{r})=\exp \left(i \boldsymbol{k}_{\|} \cdot \boldsymbol{r}\right) \varphi_{\boldsymbol{k}_{\|}, E}^{\mathrm{N}}(z)$ in the nonmagnetic layer $(z>0)$ become

$$
\begin{aligned}
\varphi_{\boldsymbol{k}_{\|}, E}^{\mathrm{N}}(0+) & =\varphi_{\boldsymbol{k}_{\|}, E}^{\mathrm{F}}(0-), \\
\frac{\partial \varphi_{\boldsymbol{k}_{\|}, E}^{\mathrm{N}}(0+)}{\partial z} & =\frac{\partial \varphi_{\boldsymbol{k}_{\|}, E}^{\mathrm{F}}(0-)}{\partial z}+\frac{2 m}{\hbar^{2}} \hat{V}_{\mathrm{int}} \varphi_{\boldsymbol{k}_{\|}, E}(0) .
\end{aligned}
$$

After some algebra, the full scattering matrix can be obtained for each transport channel which may be parametrized by the in-plane momentum $\boldsymbol{k}_{\|}$and the energy $E$. The full scattering matrix is presented in Appendix B. Here we present only the reflection matrix $\hat{r}_{\boldsymbol{k}_{\|}, E}$ for an eigenstate incident from the nonmagnetic layer, which reads

$$
\begin{aligned}
\hat{r}_{\boldsymbol{k}_{\|}, E} & =\left(k_{z} \hat{1}+\hat{\kappa}_{z, \boldsymbol{m}}+i \hat{\alpha}_{\mathrm{int}}\right)^{-1}\left(k_{z} \hat{1}-\hat{\kappa}_{z, \boldsymbol{m}}-i \hat{\alpha}_{\mathrm{int}}\right), \\
\hat{\kappa}_{z, \boldsymbol{m}} & =\frac{k_{z}^{\uparrow}+k_{z}^{\downarrow}}{2} \hat{1}+\frac{k_{z}^{\uparrow}-k_{z}^{\downarrow}}{2} \hat{\boldsymbol{\sigma}} \cdot \boldsymbol{m},
\end{aligned}
$$

where $k_{z}=\sqrt{2 m\left(E-E_{\|}\right)} / \hbar$ is the out-of-plane (along $z$ ) crystal momentum of the wave function incident from the nonmagnetic layer, $k_{z}^{\sigma}=\sqrt{2 m\left(E-E_{\|}+\sigma J_{\text {ex }}\right)} / \hbar$ is the out-of-plane crystal momentum of the wave function in the ferromagnetic layer with spin $\sigma(=\uparrow, \downarrow)$, and $\hat{\alpha}_{\text {int }}=$ $\left(2 m / \hbar^{2}\right) \hat{V}_{\text {int }}$. Here $E_{\|}=\left(\hbar^{2} / 2 m\right) \boldsymbol{k}_{\|}^{2}$ is the in-plane kinetic energy.

For further calculation, one needs to specify the form of $\hat{V}_{\text {int }}$. One option is to take the Rashba-type interfacial spinmomentum coupling, $\hat{V}_{\text {int }}=\beta^{\text {Rashba }} \hat{\boldsymbol{\sigma}} \cdot \boldsymbol{k}_{\|} \times \boldsymbol{z}$, where $z$ is the unit vector along the out-of-plane direction. Since this choice of $\hat{V}_{\text {int }}$ introduces the effective interfacial field $\beta^{\text {Rashba }} \boldsymbol{k}_{\|} \times \boldsymbol{z}$ that is not collinear to $\boldsymbol{m}$, it induces the spin nonconservation at the interface. This choice has been studied [21] in the context of the spin-memory loss. Here we take a different form of $\hat{V}_{\text {int }}$,

$$
\begin{aligned}
\hat{V}_{\mathrm{int}}= & V_{0} \hat{\sigma}_{0}+V_{\text {filter }} \hat{\boldsymbol{\sigma}} \cdot \boldsymbol{m} \\
& +\left[V_{\mathrm{sf}}^{*}|\boldsymbol{m} \uparrow\rangle\left\langle\boldsymbol{m} \downarrow\left|+V_{\mathrm{sf}}\right| \boldsymbol{m} \downarrow\right\rangle\langle\boldsymbol{m} \uparrow|\right],
\end{aligned}
$$

where $|\boldsymbol{m} \uparrow\rangle$ and $|\boldsymbol{m} \downarrow\rangle$ denote the spin states along the $+\boldsymbol{m}$ and $\boldsymbol{- m}$ directions, respectively. Note that $\hat{V}_{\text {int }}$ contains three different terms. The first term with the strength $V_{0}$ denotes the spin-independent interfacial potential, and the second term with the strength $V_{\text {filter }}$ is the interfacial spin-filtering potential. These two terms conserve the spin component along the magnetization direction $\boldsymbol{m}$. On the other hand, the third term with the strength $\left|V_{\mathrm{sf}}\right|$ induces the spin component along $\boldsymbol{m}$ to be flipped. Thus the third term amounts to the interfacial spin-flip potential. To further understand the physical implications of $\hat{V}_{\text {int }}$ in Eq. (9), it is useful to introduce the polar angle $\theta$ and the azimuthal angle $\phi$ to specify the direction of $\boldsymbol{m}=(\sin \theta \cos \phi, \sin \theta \sin \phi, \cos \theta)$. We take the two-component spinor representation $|\boldsymbol{m} \uparrow\rangle=\left(\cos (\theta / 2) e^{-i \phi / 2}, \sin (\theta / 2) e^{i \phi / 2}\right)^{\mathrm{T}} \quad$ and $\quad|\boldsymbol{m} \downarrow\rangle=$ $\left(-\sin (\theta / 2) e^{-i \phi / 2}, \cos (\theta / 2) e^{i \phi / 2}\right)^{\mathrm{T}} . \hat{V}_{\text {int }}$ then reads

$$
\hat{V}_{\text {int }}=V_{0} \hat{\sigma}_{0}+V_{\text {filter }} \hat{\boldsymbol{\sigma}} \cdot \boldsymbol{m}+\left[\operatorname{Re}\left(V_{\mathrm{sf}}\right) \hat{\boldsymbol{\sigma}} \cdot \overrightarrow{\boldsymbol{\theta}}+\operatorname{Im}\left(V_{\mathrm{sf}}\right) \hat{\boldsymbol{\sigma}} \cdot \overrightarrow{\boldsymbol{\phi}}\right],
$$

where $\overrightarrow{\boldsymbol{\theta}}=(\cos \theta \cos \phi, \cos \theta \sin \phi,-\sin \theta)$ and $\overrightarrow{\boldsymbol{\phi}}=$ $(-\sin \phi, \cos \phi, 0)$ are the unit vectors along the $\theta$ and $\phi$ directions, respectively. Thus the effective interfacial field $\left(\boldsymbol{V}_{\text {int }}\right)$ becomes

$$
\boldsymbol{V}_{\text {int }}=V_{\text {filter }} \boldsymbol{m}+\operatorname{Re}\left(V_{\text {sf }}\right) \overrightarrow{\boldsymbol{\theta}}+\operatorname{Im}\left(V_{\text {sf }}\right) \overrightarrow{\boldsymbol{\phi}},
$$

which deviates from $\boldsymbol{m}$. $\hat{V}_{\text {int }}$ with general $V_{\text {sf }}$ may be regarded as an interface potential for a bilayer system with homogeneous scatterers with the spin-orbit coupling that causes the spin-flip scattering at the interface. In this case, the interfacial 


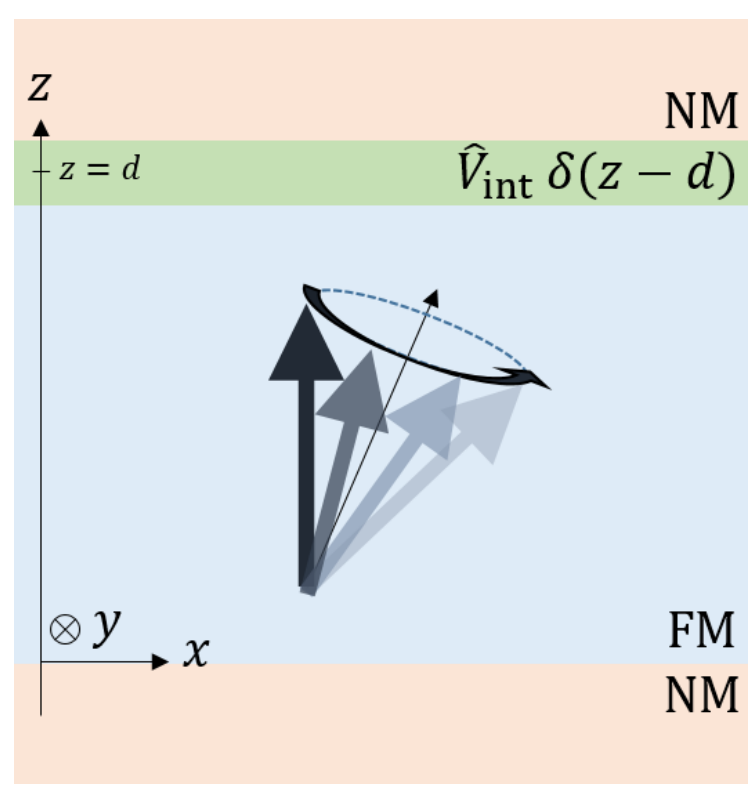

FIG. 2. Schematic diagram of a ferromagnetic metal|nonmagnetic metal|ferromagnetic metal trilayer $(\mathrm{N}|\mathrm{F}| \mathrm{N})$ with the interfacial spin-flip potential at the $\mathrm{F} \mid \mathrm{N}$ interface $(z=d)$.

spin-flip potential in Eq. (9) can be described by the ElliottYafet-like mechanism [37], and the spin of the conduction electron can be flipped at the interface after the scattering from the impurities. An atomically thin layer that consists of heavy metals may play a role similar to that of the interfacial Elliott-Yafet-like scattering in our model. Spin-orbit coupling of the ultrathin heavy metal layer between the ferromagnetic metal and the normal metal (Fig. 2) can break the spin conservation and make the spin of the conduction electron be flipped at the interface, which can be one of the the origins of the interfacial spin-flip potential [Eq. (10)]. Alternatively, when $V_{\text {sf }}$ is a pure real number, the effective field deviates from the magnetization direction towards the $\pm z$ direction, and thus $\hat{V}_{\text {int }}$ in Eq. (10) may be regarded as an interfacial potential with the strong interfacial magnetic anisotropy. Some materials (such as $\mathrm{Pt} /[\mathrm{Co} / \mathrm{Ni}]_{2}$ on the $\mathrm{MoS}_{2}$ underlayer [38], $\mathrm{Ir} / \mathrm{Co} / \mathrm{Pt}$ multilayers [39], and $\mathrm{Fe} / \mathrm{III}-\mathrm{V}$ nitride thin films [40]) are known to have a giant perpendicular magnetic anisotropy at their interfaces. The effective magnetization direction at those interfaces may deviate from the bulk magnetization direction $\boldsymbol{m}$ due to their strong interfacial magnetic anisotropy towards the $\pm z$ direction. Such effective magnetization at the interface satisfies the condition that the interfacial spin-flip potential requires in our model. Also, some of the topological materials may be candidates for the interfacial spin-flip potential. Recently, some ferromagnetic/topological insulator heterostructures (e.g., topological insulator/nonmagnet/CoFe heterostructure such as $\mathrm{Bi}_{2} \mathrm{Se}_{3} / \mathrm{Ta} / \mathrm{CoFe}$ and $\mathrm{Bi}_{2} \mathrm{Te}_{3} / \mathrm{Al} / \mathrm{CoFe}$ ) are known to make a surface magnetic anisotropy induced by their topological surface states [41]. The conducting surface states allow the pumping current of the charge, and the momentum-locked topological surface states introduce the surface magnetic anisotropy. In this respect, the surface anisotropy stemming from the topological surface states can also play a similar role of the interfacial spin-flip potential in our model.

\section{2. $N|\boldsymbol{F} \delta| N$ trilayer system with the interfacial spin-flip potential}

Figure 2 shows the $\mathrm{N}|\mathrm{F} \delta| \mathrm{N}$ trilayer system, where the symbol $\delta$ at the $\mathrm{F} \mid \mathrm{N}$ interface $(z=d)$ denotes that this interface hosts the interfacial spin-flip potential. This system has the standard configuration of the spin pumping effect [1] except that there is a spin-flip potential at an interface, which we take to be the $\mathrm{F} \mid \mathrm{N}$ interface at $z=d$ without loss of generality.

To model this $\mathrm{N}|\mathrm{F} \delta| \mathrm{N}$ trilayer system, we take the following Hamiltonian

$$
\hat{H}=\frac{\hat{p}^{2}}{2 m}+J_{\mathrm{ex}} \Theta(z) \Theta(d-z) \hat{\boldsymbol{\sigma}} \cdot \boldsymbol{m}+\hat{V}_{\mathrm{int}} \delta(z-d) .
$$

To study the the spin pumping effect, we need to calculate the scattering matrix for this Hamiltonian. By concatenating the transfer matrices of the lower bilayer $(\mathrm{N} \mid \mathrm{F})$ and the upper bilayer $(\mathrm{F} \delta \mid \mathrm{N})$, one can obtain the full scattering matrix of the trilayer system (Appendix C). For example, the transmission matrix $\hat{t}_{\boldsymbol{k}_{\|}, E}$ reads

$$
\begin{aligned}
\hat{t}_{\boldsymbol{k}_{\|}, E}= & {\left[\left(1-\frac{\hat{\alpha}_{\mathrm{int}}}{2 i k_{z}}\right) \cos \left(\hat{\kappa}_{z, \boldsymbol{m}} d\right)\right.} \\
& \left.-\left(k_{z}{ }^{2}+\hat{\kappa}_{z, \boldsymbol{m}}^{2}+i k_{z} \hat{\alpha}_{\mathrm{int}}\right) \frac{i \sin \left(\hat{\kappa}_{z, \boldsymbol{m}} d\right)}{2 k_{z} \hat{\kappa}_{z, \boldsymbol{m}}}\right]^{-1} e^{-i k_{z} d} .
\end{aligned}
$$

\section{Pumped charge current}

We apply the formula [Eqs. (1) and (2)] to calculate the pumped current from the scattering matrix. We obtain

$$
\begin{gathered}
I_{2, \text { charge }}^{\text {pump }}=\boldsymbol{G}_{2, \text { mix }}^{\text {pump }} \cdot \boldsymbol{f} \\
\left(\boldsymbol{I}_{2, \text { spin }}^{\text {pump }}\right)_{\mu}=\left(\hat{\mathcal{G}}_{2, \text { mix }}^{\text {pump }}\right)_{\mu \nu} f_{\nu},
\end{gathered}
$$

where $\boldsymbol{f}=[\hbar \omega /(2 e)] \boldsymbol{n}$, and $\boldsymbol{n}$ is the direction of the precession axis. Charge-mixing conductance $\left(\boldsymbol{G}_{l, \text { mix }}^{\text {pup }}\right)$ and generalized spin-mixing conductance tensors $\left(\hat{\mathcal{G}}_{l, \text { mix }}^{\mu, \text { pump }}\right)$ read as follows:

$$
\begin{aligned}
\boldsymbol{G}_{l, \text { mix }}^{\mathrm{pump}} & =G_{0} \sum_{n m l^{\prime}} \operatorname{Tr}\left[\left(\hat{s}_{m n, l l^{\prime}}^{\dagger}, \hat{s}_{m n, l l^{\prime}}\right) \hat{\boldsymbol{\sigma}}\right], \\
\left(\hat{\mathcal{G}}_{l, \mathrm{mix}}^{\mathrm{pump}}\right)_{\mu \nu} & =\frac{e}{4 \pi} \sum_{n m l^{\prime}} \operatorname{Tr}\left[\left(\hat{s}_{m n, l l^{\prime}}, \hat{s}_{m n, l l^{\prime}}^{\dagger} \hat{\sigma}_{\mu}\right) \hat{\sigma}_{v}\right] .
\end{aligned}
$$

Here $G_{0}=e^{2} / h$ is the conductance quantum, and $\hat{s}_{m n, l l^{\prime}}$ is the scattering matrix for instantaneous $\boldsymbol{m}$. Therefore $\boldsymbol{G}_{l, \text { mix }}^{\text {pump }}$ and $\left(\hat{\mathcal{G}}_{l, \text { mix }}^{\text {pum }}\right)_{\mu \nu}$ depend on instantaneous $\boldsymbol{m}$. We emphasize that Eq. (15a) does not vanish in general and thus the pumped current contains not only a spin current but also a charge current for general spin-nonconserving interfaces with the interfacial spin-flip potential. This is in clear contrast to spin-conserving interfaces, for which the pumped charge current vanishes since the unitary condition of the scattering matrix [Eq. (A2)] forces $\boldsymbol{G}_{l, \text { mix }}^{\text {pump }}$ to vanish. In addition, the diagonal and offdiagonal parts of $\left(\hat{\mathcal{G}}_{l, \text { mix }}^{\text {pump }}\right)_{\mu \nu}$ reduce [20,32] respectively to the original real and imaginary parts of the spin-mixing conductance at spin-conserving interfaces, and thus $\boldsymbol{I}_{2 \text {,spin }}^{\text {pump }}$ reproduces the pure spin pumping current [Eq. (4)].

We calculate the pumped charge current for the trilayer structure (Fig. 2) with the interfacial spin-flip potential 
[Eq. (10)], which reads

$$
I_{2, \text { charge }}^{\text {pump }}=\frac{e}{2 \pi}\left[\omega_{\theta} \operatorname{Im}\left(G_{2, \text { mix }}^{\text {pump }}\right)-\omega_{\phi} \sin \theta \operatorname{Re}\left(G_{2, \text { mix }}^{\text {pump }}\right)\right],
$$

where $\omega_{\theta(\phi)}=d \theta / d t(d \phi / d t)$, and the charge-mixing conductance $\left(G_{2, \text { mix }}^{\text {pump }}\right)$ becomes as follows:

$$
G_{2, \text { mix }}^{\text {pump }}=G_{0} \sum_{n m}\left[r_{m n}^{\uparrow} r_{m n}^{+*}+r_{m n}^{\downarrow *} r_{m n}^{-}+t_{m n}^{\prime \uparrow} t_{m n}^{\prime * *}+t_{m n}^{\prime \downarrow *} t_{m n}^{\prime-}\right] .
$$

Note that all terms in Eq. (17) contain the off-diagonal scattering elements $\left(r_{m n}^{+(-)}, t_{m n}^{\prime+(-)}\right)$ and thus $G_{2 \text {,mix }}^{\text {charge }}$ vanishes when $V_{\mathrm{sf}}=0$. To simplify the model calculation, we assume that $V_{\mathrm{sf}}$ is small and retain only the linear contributions of $V_{\text {sf }}$ to $I_{2, \text { charge }}^{\text {pump }}$ to obtain

$$
\begin{gathered}
I_{2, \text { charge }}^{\text {pump }}=\frac{e}{2 \pi}\left[\operatorname{Re}\left(G_{\text {int }}^{\mathrm{cp}}\right) \boldsymbol{m} \times \dot{\boldsymbol{m}}-\operatorname{Im}\left(G_{\text {int }}^{\mathrm{cp}}\right) \dot{\boldsymbol{m}}\right] \cdot \boldsymbol{\alpha}_{\mathrm{int}}, \\
\boldsymbol{\alpha}_{\mathrm{int}}=\frac{2 m}{\hbar^{2}} \boldsymbol{V}_{\mathrm{int}},
\end{gathered}
$$

where the interfacial charge pumping conductance $\left(G_{\text {int }}^{\mathrm{cp}}\right)$ reads

$$
\begin{aligned}
G_{\text {int }}^{\text {cp }}= & \sum_{k_{\|}} \frac{4 k_{z}^{2}}{\left|D_{\uparrow}\right|^{2}\left|D_{\downarrow}\right|^{2}}\left[k_{z}^{\downarrow}\left(k_{z}^{\uparrow^{2}}-k_{z}^{2}\right) \sin \left(k_{z}^{\uparrow} d\right)\right. \\
& \left.+k_{z}^{\uparrow}\left(k_{z}^{2}-k_{z}^{\downarrow}\right) \sin \left(k_{z}^{\downarrow} d\right)\right] \\
& \times\left[k_{z}^{\uparrow} k_{z}^{\downarrow}\left[\cos \left(k_{z}^{\downarrow} d\right)-\cos \left(k_{z}^{\uparrow} d\right)\right]\right. \\
& \left.+i k_{z}\left[k_{z}^{\uparrow} \sin \left(k_{z}^{\downarrow} d\right)+k_{z}^{\downarrow} \sin \left(k_{z}^{\uparrow} d\right)\right]\right], \\
D_{\sigma}= & 2 i k_{z} k_{z}^{\sigma} \cos \left(k_{z}^{\sigma} d\right)+\left(k_{z}^{2}+k_{z}^{\sigma 2}\right) \sin \left(k_{z}^{\sigma} d\right) .
\end{aligned}
$$

Here $G_{\text {int }}^{\mathrm{cp}}$ is evaluated in the limit $\boldsymbol{V}_{\text {int }}=0$ and is independent of the direction of $\boldsymbol{m} . G_{\text {int }}^{\mathrm{cp}}$ vanishes when $k_{z}=k_{z}^{\uparrow}=k_{z}^{\downarrow}$, which is the case that exchange interaction of the ferromagnet vanishes $\left(J_{\mathrm{ex}} \rightarrow 0\right)$. This indicates that not only the spin-flip potential at the interface $\left(\hat{V}_{\text {int }}\right)$ but also the exchange interaction of the ferromagnet $\left(J_{\mathrm{ex}}\right)$ are responsible for the charge pumping. $G_{\text {int }}^{\mathrm{cp}}$ also vanishes when $d \rightarrow 0$, which is the case that the $\mathrm{N} \mid \mathrm{F} \delta \mathrm{N}$ trilayers become $\mathrm{N} \mid \delta \mathrm{N}$ bilayers. In this case, $\mathrm{N} \mid \delta \mathrm{N}$ bilayers restore the inversion symmetry, and the pumped charge current trivially vanishes.

\section{DISCUSSION}

\section{A. Connection with magnetoelectronic circuit theory}

In this section, we discuss the connection between the adiabatic pumping theory and the magnetoelectronic circuit theory. In spin-conserving systems, this connection is well understood; the adiabatic quantum pumping theory can be mapped to a special case of the magnetoelectronic circuit theory [2]. In spin-nonconserving systems, however, it is not clear how this type of connection appears. In Sec. III A 2, we examine this connection. In Sec. III A 1, we summarize the connection in spin-conserving systems [2] as a preparation for the discussion in spin-nonconserving systems, Sec. III A 2.

\section{Connection in spin-conserving systems}

The charge transport in the mesoscopic regime can be described by the Landauer-Buttiker formalism. In this regime, the current of an electric circuit can be formulated with the scattering matrix and distribution function of each terminal. This formalism can be extended to the spin space in a way similar to that in which the adiabatic quantum pumping theory was extended to the spin pumping theory. This extended version of the circuit theory, called magnetoelectronic circuit theory, can deal with not only the charge current but also the spin current. According to the magnetoelectronic circuit theory, the current tensor in terminal 2 reads in two-terminal systems as follows:

$$
\hat{I}_{2}^{\text {circuit }}=G_{0} \sum_{n m}\left[\hat{t}_{m n}^{\prime} \hat{f}_{1}\left(\hat{t}_{m n}^{\prime}\right)^{\dagger}+\hat{r}_{m n} \hat{f}_{2}\left(\hat{r}_{m n}\right)^{\dagger}-\delta_{m n} \hat{f}_{2}\right],
$$

where $\hat{f}_{1}$ and $\hat{f}_{2}$ are distribution functions of terminal 1 and terminal 2 , respectively, and the reflection matrix $\left(\hat{r}_{m n}\right)$ and the transmission matrix $\left(\hat{t}_{m n}^{\prime}\right)$ are already introduced in the discussion of the spin pumping theory [Eq. (3)].

The magnetoelectronic circuit theory deals with the charge and spin currents induced by static biases $\hat{f}_{1}$ and $\hat{f}_{2}$. So its realm of applicability differs from that of the spin pumping theory, which describes the currents induced by slow precession of the magnetization. However, it turns out that they are closely related. For the spin-conserving system, $\hat{I}_{2}^{\text {circuit }}$ in Eq. (22) reduces to

$$
\begin{aligned}
I_{2, \text { charge }}^{\text {circuit }} & =G_{2}^{\text {cc }} \Delta f_{0}+\boldsymbol{G}_{2}^{\mathrm{cs}} \cdot \boldsymbol{\Delta} \boldsymbol{f}_{\boldsymbol{s}}, \\
\boldsymbol{I}_{2, \text { spin }}^{\text {circuit }} & =\boldsymbol{G}_{2}^{\mathrm{sc}} \Delta f_{0}+\mathcal{G}_{2}^{\mathrm{ss}} \cdot \boldsymbol{\Delta} \boldsymbol{f}_{\boldsymbol{s}}-\mathcal{G}_{2}^{\text {mix }} \cdot \boldsymbol{f}_{2, s},
\end{aligned}
$$

where $\quad \Delta f_{0}=\operatorname{Tr}\left[\hat{\sigma}_{0}\left(\hat{f}_{1}-\hat{f}_{2}\right)\right] / 2, \quad\left(\boldsymbol{\Delta} \boldsymbol{f}_{s}\right)_{\mu}=\operatorname{Tr}\left[\hat{\sigma}_{\mu}\left(\hat{f}_{1}-\right.\right.$ $\left.\left.\hat{f}_{2}\right)\right] / 2$, and $\left(\boldsymbol{f}_{2, s}\right)_{\mu}=\operatorname{Tr}\left[\hat{\sigma}_{\mu} \hat{f}_{2}\right] / 2$. Here $G_{2}^{\text {cc }}, \boldsymbol{G}_{2}^{\text {ss }}, \boldsymbol{G}_{2}^{\text {sc }}, \mathcal{G}_{2}^{\text {ss }}$, and $\mathcal{G}_{2}^{\text {mix }}$ are related to the majority/minority conductance $\left(G^{\uparrow / \downarrow}\right)$ and the spin-mixing conductance $\left(g^{\uparrow \downarrow}\right) ; G_{2}^{\mathrm{cc}}=\left(G^{\uparrow}+G^{\downarrow}\right)$, $\boldsymbol{G}_{2}^{\mathrm{cs}}=\boldsymbol{G}_{2}^{\mathrm{sc}}=\left(G^{\uparrow}-G^{\downarrow}\right) \boldsymbol{m}, \quad \mathcal{G}_{2}^{\mathrm{ss}}=\left(G^{\uparrow}+G^{\downarrow}\right) \boldsymbol{m} \boldsymbol{m}^{\mathrm{T}}, \quad$ and $\mathcal{G}_{2}^{\text {mix }} / 2=\operatorname{Re}\left(g^{\uparrow \downarrow}\right) \boldsymbol{m} \times(\boldsymbol{m} \times)-\operatorname{Im}\left(g^{\uparrow \downarrow}\right)(\boldsymbol{m} \times)$. We emphasize that the spin-mixing conductance $\left(g^{\uparrow \downarrow}\right)$ in the expression for $\mathcal{G}_{2}^{\text {mix }}$ is identical to the spin-mixing conductance introduced in the discussion of the spin pumping theory [Eq. (4)] in spin-conserving systems. This identity can be derived by mapping a problem with slow precession of the magnetization to a problem without precession but instead with spin biases [2].

\section{Connection in spin-nonconserving systems}

The interfacial spin-flip potential induces $\hat{r}$ and $\hat{t}^{\prime}$ to acquire off-diagonal components in spin space. In this case, the conventional magnetoelectronic circuit theory needs to be generalized.

The formula [Eq. (22)] for the current tensor remains valid even in the presence of the interfacial spin-flip potential. The generalized magnetoelectronic circuit theory $[15,20]$ uses Eq. (22) to take into account both spin-conserving scattering and spin-nonconserving scattering and obtains the charge and spin currents as follows:

$$
\begin{aligned}
I_{2, \text { charge }}^{\text {circuit }} & =\tilde{G}_{2}^{\text {cc }} \Delta f_{0}+\tilde{\boldsymbol{G}}_{2}^{\mathrm{cs}} \cdot \boldsymbol{\Delta} \boldsymbol{f}_{s}-\tilde{\boldsymbol{G}}_{2}^{\mathrm{mix}} \cdot \boldsymbol{f}_{2, s}, \\
\boldsymbol{I}_{2, \text { spin }}^{\text {circuit }} & =\tilde{\boldsymbol{G}}_{2}^{\mathrm{sc}} \Delta f_{0}+\tilde{\mathcal{G}}_{2}^{\mathrm{ss}} \cdot \boldsymbol{\Delta} \boldsymbol{f}_{s}-\tilde{\mathcal{G}}_{2}^{\mathrm{mix}} \cdot \boldsymbol{f}_{2, s} .
\end{aligned}
$$

Note that Eq. (24) is very similar to Eq. (23) except that Eq. (24a) contains a third term that does not have a counterpart 
in Eq. (23a). We briefly discuss the connection between the third term and the charge pumping. By the way, Refs. [15,20] utilized Eq. (24) to examine properties of the spin transport phenomena in spin-nonconserving systems. For instance, it was emphasized that whereas $\mathcal{G}_{2}^{\text {mix }}$ can be expressed as a sum of two terms proportional to $\boldsymbol{m} \times$ and $\boldsymbol{m} \times(\boldsymbol{m} \times), \tilde{\mathcal{G}}_{2}^{\text {mix }}$ contains the third term that is proportional to $\boldsymbol{m} \boldsymbol{m}^{\mathrm{T}}$. It was shown that the third term can give rise to the spin-memory loss [13].

Reference [20] provides an explicit formula for $\tilde{\boldsymbol{G}}_{2}^{\text {mix }}$. We apply the formula to the $\mathrm{N}|\mathrm{F}| \delta \mathrm{N}$ trilayers in Sec. II B and verify the following relation,

$$
\boldsymbol{G}_{2, \text { mix }}^{\text {pump }}=-\tilde{\boldsymbol{G}}_{2}^{\text {mix }},
$$

where $\boldsymbol{G}_{2 \text {,mix }}^{\text {pump }}$ is the charge-mixing conductance that determines the charge current pumped by the precessing magnetization [Eq. (16)]. Equation (25) thus implies that the charge pumping in spin-nonconserving systems may be related to the generalized magnetoelectronic circuit theory.

This relation can be understood by applying a similar mapping that has been used to understand the relation between the spin pumping and the conventional magnetoelectronic circuit theory in spin-conserving systems [2]. The problem of a precessing magnetization in Sec. II B can be mapped to a problem without magnetization precession but instead with biases by applying a proper unitary transformation $\hat{U}$. For instance, when the magnetization $\boldsymbol{m}$ precesses around the axis $\boldsymbol{n}$ with the angular frequency $\omega$, we may take $\hat{U}=\exp [-i \hat{\boldsymbol{\sigma}}$. $\left.\boldsymbol{n} \omega\left(t-t_{0}\right) / 2\right]$. Then for the $\mathrm{N}|\mathrm{F}| \delta \mathrm{N}$ trilayers in Sec. II B, the unitary transformation by $\hat{U}$ maps the Hamiltonian $H[\boldsymbol{m}(t)]$ for the precessing magnetization $\boldsymbol{m}(t)$ to the time-independent Hamiltonian $H\left[\boldsymbol{m}\left(t=t_{0}\right)\right]$ :

$$
H[\boldsymbol{m}(t)] \rightarrow \hat{U}^{\dagger} H[\boldsymbol{m}(t)] \hat{U}=H\left[\boldsymbol{m}\left(t=t_{0}\right)\right] .
$$

Note that the transformed Hamiltonian is the time independent and thus one may apply the generalized magnetoelectronic circuit theory [Eq. (22)] to evaluate both charge and spin currents pumped by precessing $\boldsymbol{m}(t)$. The time-independent biases $\hat{f}_{1}$ and $\hat{f}_{2}$ for the generalized magnetoelectronic circuit theory are also fixed by the transformation itself since $\hat{U}$ is time dependent, $\hat{f}_{1}=\hat{f}_{2}=\hat{f}_{\text {pump }} \equiv i(\hbar / e) \hat{U}^{\dagger}(d \hat{U} / d t)=$ $[\hbar \omega /(2 e)] \hat{\boldsymbol{\sigma}} \cdot \boldsymbol{n}$. This way, one finds that the pumped charge and spin currents at time $t=t_{0}$ are given by the current tensor:

$$
\hat{I}_{2}^{\text {pump }}=G_{0} \sum_{n m}\left[{\hat{t^{\prime}}}_{m n} \hat{f}_{\text {pump }}\left({\hat{t^{\prime}}}_{m n}\right)^{\dagger}+\hat{r}_{m n} \hat{f}_{\text {pump }}\left(\hat{r}_{m n}\right)^{\dagger}-\delta_{m n} \hat{f}_{\text {pump }}\right] \text {, }
$$

where ${\hat{t^{\prime}}}_{m n}$ and $\hat{r}_{m n}$ are the transmission and reflection amplitudes for $\boldsymbol{m}=\boldsymbol{m}\left(t_{0}\right)$, respectively. Equation (27) implies that $\hat{I}_{2}^{\text {pump }}$, which was previously evaluated by the adiabatic quantum pumping theory, can be evaluated alternatively by the generalized magnetoelectonic circuit theory. This explains the relation [Eq. (25)].

However, we remark that the relation [Eq. (25)] is not always valid for arbitrary spin-nonconserving systems. For Eq. (25) to be valid, the problem of a precessing magnetization should be mapped to a time-independent problem through a proper unitary transformation $\hat{U}$. For the $\mathrm{N}|\mathrm{F} \delta| \mathrm{N}$ trilayers in Sec. II B, such $\hat{U}$ exists. But for general spin-nonconserving systems, there is no guarantee that such $\hat{U}$ exists [29]. For example, if $\hat{V}_{\text {int }}$ in Eq. (6) is given by

$$
\hat{V}_{\text {int }}(z)=\operatorname{Re}\left(V_{\text {sf }}\right) \hat{\sigma}_{x}+\operatorname{Im}\left(V_{\text {sf }}\right) \hat{\sigma}_{y},
$$

such $\hat{U}$ does not exist. A crucial difference between $\hat{V}_{\text {int }}$ in Eq. (28) and $\hat{V}_{\text {int }}$ in Eq. (9) is that the latter is expressed in terms of $\boldsymbol{m}$ and thus is covariant with $\boldsymbol{m}$, which allows $\hat{U}$ to exist, whereas the former has its own direction $\left[\operatorname{Re}\left(V_{\mathrm{sf}}\right) \boldsymbol{x}+\right.$ $\left.\operatorname{Im}\left(V_{\text {sf }}\right) \boldsymbol{y}\right]$ and is not covariant, thus forbidding $\hat{U}$ to exist.

\section{B. Comparison with other charge-current-generating effects}

A precessing magnetization can generate a charge current or an electric voltage in various ways. In this section, we compare the pumped charge current with other mechanisms to generate a charge current from a precessing magnetization. To separate the dc-voltage-generating effects upon their mechanisms, we can divide them into two groups; one group consists of the phenomena that generate dc voltage, but does not directly result from adiabatic quantum pumping effects. For example, such a group can include the inverse spin-Hall effect (ISHE) [42], the spin-rectification (SR) effect [43], and the backflow-generating mechanism $[44,45]$. First, the ISHE is well known for the charge-current-generating effects via the spin current. Electric voltage can be generated in $\mathrm{N} \mid \mathrm{F}$ bilayers when a pumped spin current in $\mathrm{N}$ experiences the inverse spinHall effect [42]. This voltage is developed along an in-plane direction and thus differs from the electric voltage that may be generated due to the pumped charge current. To be more exact, it refers to the phenomenon that generates charge currents by converting spin currents into charge currents via the spin-orbit interaction. The induced charge current via the ISHE flows perpendicular to both the spin polarization vector and the flow direction of the spin current. The pumped charge current we are interested in, on the other hand, is generated from the precession of uniform magnetization in the presence of the interfacial spin-flip potential, and it gives the out-of-plane (along the $z$ direction) current. Therefore the pumping effect is not a spin-to-charge converting phenomenon that requires the existence of the spin currents to generate charge current, and one can classify these two effects on symmetry analysis by investigating its flowing direction. Second, the spin-rectification (SR) effect may generate dc voltage due to the coupling between a time-dependent resistance $R[\boldsymbol{M}(t)]$ and current $\boldsymbol{I}(t)$. The SR effect rectifies the microwave current in ferromagnets, and consequently, the ferromagnetic resonance (FMR) voltage contributed from the spin pumping effect is usually accompanied by that from spin rectification, in general. Fortunately, we can separate these two effects by investigating the FMR line shape [46] or by the symmetry analysis between the voltage signal of spin pumping and the SR effect $[43,47]$. Third, the back-flow mechanism $[44,45,48]$ is the secondary effect of the spin pumping effect. Due to the spin current injected into the interface from the spin pumping effect, the accumulated spin chemical potential at the interface can produce a backflow current into the ferromagnet, which is consequently responsible for the voltage drop across the interface. This makes them more difficult to distinguish by their origin, because the backflow current also becomes larger as the spin pumping effect gets stronger. If we assume a small 
interfacial spin-flip potential, we can expect that the backflow current is proportional to the spin pumping current [Eq. (14b)] and the charge pumping current is proportional to the chargemixing conductance we defined [Eq. (14a)]. Comparing the two conductances [Eqs. (14a) and (14b)] related with each effect, two phenomena can be distinguished quantitatively. The ratio between the two conductances is comparable (0.96-1.21) for a small $V_{\mathrm{sf}}\left(V_{\mathrm{sf}}=0.1 \AA \mathrm{eV}\right)$ and typical ferromagnetic materials $\left(J_{\mathrm{ex}}=1.0-1.3 \mathrm{eV}\right)$. This implies that the charge pumping current [Eq. (16)] can be comparable with the backflow current, or even be larger in a small precession angle.

The other group consists of the phenomena that are actually originated from the adiabatic quantum pumping effects. In this case, the pumping current can be originated either from the bulk or from the interface. When it is originated from the bulk, then the signal can stem from either the nonmagnetic metal layer or the ferromagnetic metal layer. The charge pumping driven by magnon due to the spin-orbit coupling of the nonmagnetic metal is studied, and it turns out that the dc voltage signal (along the in-plane direction) due to this mechanism is small because it is proportional to the second order of the precession amplitude [27]. Although charge pumping effects are also investigated in various different structures, such as magnetic tunnel junctions [23,29], graphene [49], chiral magnetization [50] with spin wave, and topological matters [51], they go beyond our formulation. In experiment, the signals will come from the total contribution of all dc-generating effects in general.

\section{CONCLUSION}

In this work, we explore the charge pumping on both sides of the adiabatic quantum pumping theory and generalized magnetoelectronic circuit theory in the presence of the ElliottYafet-like spin-flip potential [Eq. (10)] at the interface. Using the adiabatic quantum pumping theory, we show the analytic expression for the pumped charge current in terms of the scattering matrix, and we find that the pumped charge current, which conventionally vanishes at spin-conserving interfaces, does not vanish in general spin-nonconserving interfaces. The pumped charge current may induce a voltage difference between ferromagnets and their adjacent materials, and this voltage difference can make the experimental analysis of spin pumping complicated. Our results can provide a tool to calculate the pumped charge current.

The conventional spin pumping theory is known to be directly related to magnetoelectronic circuit theory in the case of the spin-conserving interface. Recently, it has also been reported that the concept of the spin-dependent conductances can be generalized to the conductance tensor [Eq. (24)]. We find that the similar link between the two theories can also be extended to the pumped charge current in our spinnonconserving system. We show that the pumped charge current originated from the Elliott-Yafet-like spin-flip potential can be directly related to the extended magnetoelectronic circuit theory, and we present that the expression of the pumped charge current can be rewritten in terms of the generalized conductance tensors. Also, we report the condition of a nonapplicable case that may not show the generalized connection between the two theories.

\section{ACKNOWLEDGMENTS}

This work was supported by the National Research Foundation of Korea (NRF) (Grants No. 2018R1A5A6075964 and No. 2020R1A2C2013484).

\section{APPENDIX A: UNITARY CONDITION}

The scattering matrix should obey the unitary condition in the spin-nonconserving interface, since the charge-current flux should always be conserved regardless of whether the spin angular momentum is conserved or not at the interface. The unitary condition reads

$$
S S^{\dagger}=S^{\dagger} S=I_{N+M},
$$

where $S$ is the scattering matrix and $I_{N+M}$ denotes the identity matrix of size $N+M$ in the transporting channel space. The unitary condition [Eq. (A1)] gives the following identities which read as follows:

$$
\begin{aligned}
& \sum_{m n}\left[\hat{r}_{m n}\left(\hat{r}_{m n}\right)^{\dagger}+\hat{t}_{m n}^{\prime}\left(\hat{t}_{m n}^{\prime}\right)^{\dagger}\right]=N \hat{\sigma}_{0}, \\
& \sum_{m n}\left[\hat{t}_{m n}\left(\hat{t}_{m n}\right)^{\dagger}+\hat{r}_{m n}^{\prime}\left(\hat{r}_{m n}^{\prime}\right)^{\dagger}\right]=M \hat{\sigma}_{0}, \\
& \sum_{m n}\left[\left(\hat{r}_{m n}\right)^{\dagger} \hat{r}_{m n}+\left(\hat{t}_{m n}\right)^{\dagger} \hat{t}_{m n}\right]=N \hat{\sigma}_{0}, \\
& \sum_{m n}\left[\left(\hat{t}_{m n}^{\prime}\right)^{\dagger} \hat{t}_{m n}^{\prime}+\left(\hat{r}_{m n}^{\prime}\right)^{\dagger} \hat{r}_{m n}^{\prime}\right]=M \hat{\sigma}_{0},
\end{aligned}
$$

where $N$ and $M$ are the number of the transport channel of terminal 2 and terminal 1, respectively. The transmission matrices $\left(\hat{t}_{m n}^{\prime}, \hat{t}_{m n}\right)$ and the reflection matrices $\left(\hat{r}_{m n}^{\prime}, \hat{r}_{m n}\right)$ are defined the same as they are in the main body of this paper.

\section{APPENDIX B: DERIVATION OF THE SCATTERING MATRICES FOR BILAYER STRUCTURE (F $\delta \mid \mathbf{N})$}

Here, we define the scattering states for the bilayer structure (Fig. 1) with a precessing ferromagnet, and then we derive the scattering matrix explicitly. The scattering states $\psi_{\boldsymbol{k}_{\|}, E}^{\mathrm{F}}$ in the ferromagnet $(z<0)$ and $\psi_{\boldsymbol{k}_{\|}, E}^{\mathrm{N}}$ in the nonmagnetic metal $(z>0)$ can be written as

$$
\begin{aligned}
\psi_{\boldsymbol{k}_{\|}, E}^{\mathrm{F}}(\boldsymbol{r}) & =\exp \left(i \boldsymbol{k}_{\|} \cdot \boldsymbol{r}\right) \hat{U} \sqrt{\frac{k_{z}}{\hat{\kappa}_{z}}}\left(e^{i \hat{\kappa}_{z} z} A_{1}+e^{-i \hat{\kappa}_{z} z} B_{1}\right), \\
\psi_{\boldsymbol{k}_{\|}, E}^{\mathrm{N}}(\boldsymbol{r}) & =\exp \left(i \boldsymbol{k}_{\|} \cdot \boldsymbol{r}\right)\left(e^{i k_{z} z} A_{2}+e^{-i k_{z} z} B_{2}\right), \\
\hat{\kappa}_{z} & =\frac{k_{z}^{\uparrow}+k_{z}^{\downarrow}}{2} \hat{1}+\frac{k_{z}^{\uparrow}-k_{z}^{\downarrow}}{2} \hat{\sigma}_{z},
\end{aligned}
$$

where $\boldsymbol{k}_{\|}$denotes the in-plane crystal momentum, which is perpendicular to the propagating direction $z$. $\hat{U}$ is the rotation matrix in the spin space around the direction of the precession axis. 
Continuity of the scattering states at the interface $(z=0)$ requires

$$
\begin{aligned}
& \psi_{\mathrm{N}}(0+)=\psi_{\mathrm{F}}(0-), \\
& A_{2}+B_{2}=\hat{U} \sqrt{\frac{k_{z}}{\hat{\kappa}_{z}}}\left(A_{1}+B_{1}\right) .
\end{aligned}
$$

Due to the interfacial spin-flip potential, this gives the condition of the derivative of the scattering states, which reads as follows:

$$
\begin{aligned}
& \psi_{\mathrm{N}}^{\prime}(0+)-\psi_{\mathrm{F}}^{\prime}(0-)=\hat{\alpha}_{\mathrm{int}} \psi(0), \\
& i k_{z}\left(A_{2}-B_{2}\right)-\hat{U} \sqrt{\frac{k_{z}}{\hat{\kappa}_{z}}}\left(i \hat{\kappa}_{z}\right)\left(A_{1}-B_{1}\right) \\
& \quad=\hat{\alpha}_{\mathrm{int}} \hat{U} \sqrt{\frac{k_{z}}{\hat{\kappa}_{z}}}\left(A_{1}+B_{1}\right) .
\end{aligned}
$$

Matching the boundary conditions given by [Eq. (B2)] and [Eq. (B3)], we can find the transfer matrix for the bilayer structure with interfacial potential, which reads as follows:

$$
\begin{aligned}
& \left(\begin{array}{cc}
\hat{U} \sqrt{\frac{k_{z}}{\hat{\kappa}_{z}}} & \hat{U} \sqrt{\frac{\bar{k}_{z}}{\hat{\kappa}_{z}}} \\
\hat{U} \sqrt{\frac{\hat{\kappa}_{z}}{k_{z}}}+\frac{1}{i k_{z}} \hat{U} \hat{\alpha}_{\mathrm{int}} & -\hat{U} \sqrt{\frac{\hat{\kappa}_{z}}{k_{z}}}+\frac{1}{i k_{z}} \hat{U} \hat{\alpha}_{\mathrm{int}}
\end{array}\right)\left(\begin{array}{l}
A_{1} \\
B_{1}
\end{array}\right) \\
& =\left(\begin{array}{cc}
1 & 1 \\
1 & -1
\end{array}\right)\left(\begin{array}{l}
A_{2} \\
B_{2}
\end{array}\right),
\end{aligned}
$$

$$
\begin{aligned}
\left(\begin{array}{l}
A_{2} \\
B_{2}
\end{array}\right)= & (\hat{1} \otimes \hat{U})\left(\begin{array}{ll}
k_{z}+\hat{\kappa}_{z}-i \hat{\alpha}_{\mathrm{int}}^{z} & k_{z}-\hat{\kappa}_{z}-i \hat{\alpha}_{\mathrm{int}}^{z} \\
k_{z}-\hat{\kappa}_{z}+i \hat{\alpha}_{\mathrm{int}}^{z} & k_{z}+\hat{\kappa}_{z}+i \hat{\alpha}_{\mathrm{int}}^{z}
\end{array}\right) \\
& \times \frac{1}{\sqrt{k_{z} \hat{\kappa}_{z}}}\left(\begin{array}{l}
A_{1} \\
B_{1}
\end{array}\right), \\
= & \left(\begin{array}{ll}
M_{11} & M_{12} \\
M_{21} & M_{22}
\end{array}\right)=M\left(\begin{array}{l}
A_{1} \\
B_{1}
\end{array}\right) .
\end{aligned}
$$

Here, the scattering matrix can be written in terms of the elements of the transfer matrix (B5), which reads as

$$
\begin{aligned}
S & =\left(\begin{array}{cc}
M_{12} M_{22}^{-1} & M_{11}-M_{12} M_{22}^{-1} M_{21} \\
M_{22}^{-1} & -M_{22}^{-1} M_{21}
\end{array}\right), \\
& =\left(\begin{array}{cc}
\hat{U} r_{c} \hat{U}^{\dagger} & \hat{U} t^{\prime}{ }_{c} \\
t_{c} \hat{U}^{\dagger} & r^{\prime}{ }_{c}
\end{array}\right),
\end{aligned}
$$

where $s_{\mathrm{c}}\left(s=r, t, t^{\prime}, r^{\prime}\right)$ is the scattering matrix when $\boldsymbol{m}=\boldsymbol{z}$. The explicit expressions for the reflection and transmission matrices in [Eq. (B6b)] read as follows:

$$
\begin{aligned}
r_{\mathrm{c}} & =\left(k_{z}+\hat{\kappa}_{z}+i \hat{\alpha}_{\mathrm{int}}^{z}\right)^{-1}\left(k_{z}-\hat{\kappa}_{z}-i \hat{\alpha}_{\mathrm{int}}^{z}\right), \\
t_{\mathrm{c}}^{\prime} & =\left(k_{z}+\hat{\kappa}_{z}+i \hat{\alpha}_{\mathrm{int}}^{z}\right)^{-1} 2 \sqrt{k_{z} \hat{\kappa}_{z}}, \\
t_{\mathrm{c}} & =2 \sqrt{k_{z} \hat{\kappa}_{z}}\left(k_{z}+\hat{\kappa}_{z}+i \hat{\alpha}_{\mathrm{int}}^{z}\right)^{-1}, \\
r_{\mathrm{c}}^{\prime} & =-\sqrt{\hat{\kappa}_{z}}\left(k_{z}+\hat{\kappa}_{z}+i \hat{\alpha}_{\mathrm{int}}^{z}\right)^{-1}\left(k_{z}-\hat{\kappa}_{z}+i \hat{\alpha}_{\mathrm{int}}^{z}\right) \frac{1}{\sqrt{\hat{\kappa}_{z}}} .
\end{aligned}
$$

The scattering matrices of the $\mathrm{N} \mid \mathrm{F}$ bilayers [Eq. (B7)] reproduce the results of the scattering matrices derived by Kim et al. [22].

\section{APPENDIX C: DERIVATION OF THE TRANSFER MATRIX FOR N|F $\delta \mid N$ STRUCTURE}

In a way similar to that shown in Appendix B, we calculate the transfer matrix $\left(M_{\mathrm{III}}\right)$ for the interface between region I $(z<0)$ and region II $(0<z<d)$ and the transfer matrix $\left(M_{\text {III,II }}\right)$ for the interface between region II and region III $(z>d)$, which read as follows:

$$
\begin{aligned}
M_{\mathrm{III}, \mathrm{II}} & =(\hat{\imath} \otimes \hat{U})\left(\begin{array}{cc}
\left(k_{z}+\hat{\kappa}_{z}-i \hat{\alpha}_{\mathrm{int}}^{z}\right) e^{-i k_{z} d} e^{i \hat{\kappa}_{z} d} & \left(k_{z}-\hat{\kappa}_{z}-i \hat{\alpha}_{\mathrm{int}}^{z}\right) e^{-i k_{z} d} e^{-i \hat{\kappa}_{z} d} \\
\left(k_{z}-\hat{\kappa}_{z}+i \hat{\alpha}_{\mathrm{int}}^{z}\right) e^{i k_{z} d} e^{i \hat{\kappa}_{z} d} & \left(k_{z}+\hat{\kappa}_{z}+i \hat{\alpha}_{\mathrm{int}}^{z}\right) e^{i k_{z} d} e^{-i \hat{\kappa}_{z} d}
\end{array}\right) \frac{1}{2 \sqrt{k_{z} \hat{\kappa}_{z}}}, \\
M_{\mathrm{IIII}} & =\frac{1}{2 \sqrt{k_{z} \hat{\kappa}_{z}}}\left(\begin{array}{cc}
k_{z}+\hat{\kappa}_{z} & -\left(k_{z}-\hat{\kappa}_{z}\right) \\
-\left(k_{z}-\hat{\kappa}_{z}\right) & k_{z}+\hat{\kappa}_{z}
\end{array}\right)(\hat{1} \otimes \hat{U})^{\dagger} .
\end{aligned}
$$

Concatenating the transfer matrices [Eq. (C1)], we calculate the composite transfer matrix $\left(M_{\mathrm{III}, \mathrm{I}}\right)$ for the interface between region III and region I, which reads as follows:

$$
\begin{gathered}
M_{\mathrm{III,I}}^{\mathrm{c}}=(\hat{1} \otimes \hat{U})^{\dagger} M_{\mathrm{III}, \mathrm{II}} M_{\mathrm{II}, \mathrm{I}}(\hat{1} \otimes \hat{U}), \\
M_{\mathrm{III}, \mathrm{I}}^{\mathrm{c} 1,1}=\left[\left(1+\frac{\hat{\alpha}_{\mathrm{int}}^{z}}{2 i k_{z}}\right) \cos \left(\hat{\kappa}_{z} d\right)+\left(k_{z}^{2}+\hat{\kappa}_{z}^{2}-i k_{z} \hat{\alpha}_{\mathrm{int}}^{z}\right) \frac{i \sin \left(\hat{\kappa}_{z} d\right)}{2 k_{z} \hat{\kappa}_{z}}\right] e^{-i k_{z} d}, \\
M_{\mathrm{III}, \mathrm{I}}^{\mathrm{c} 1,2}=\left[\frac{\hat{\alpha}_{\mathrm{int}}^{z}}{2 i k_{z}} \cos \left(\hat{\kappa}_{z} d\right)-\left(k_{z}^{2}-\hat{\kappa}_{z}^{2}-i k_{z} \hat{\alpha}_{\mathrm{int}}^{z}\right) \frac{i \sin \left(\hat{\kappa}_{z} d\right)}{2 k_{z} \hat{\kappa}_{z}}\right] e^{-i k_{z} d}, \\
M_{\mathrm{III}, \mathrm{I}}^{\mathrm{c} 2,1}=\left[-\frac{\hat{\alpha}_{\mathrm{int}}^{z}}{2 i k_{z}} \cos \left(\hat{\kappa}_{z} d\right)+\left(k_{z}^{2}-\hat{\kappa}_{z}^{2}+i k_{z} \hat{\alpha}_{\mathrm{int}}^{z}\right) \frac{i \sin \left(\hat{\kappa}_{z} d\right)}{2 k_{z} \hat{\kappa}_{z}}\right] e^{i k_{z} d}, \\
M_{\mathrm{III}, \mathrm{I}}^{\mathrm{c} 2,2}=\left[\left(1-\frac{\hat{\alpha}_{\mathrm{int}}^{z}}{2 i k_{z}}\right) \cos \left(\hat{\kappa}_{z} d\right)-\left(k_{z}^{2}+\hat{\kappa}_{z}^{2}+i k_{z} \hat{\alpha}_{\mathrm{int}}^{z}\right) \frac{i \sin \left(\hat{\kappa}_{z} d\right)}{2 k_{z} \hat{\kappa}_{z}}\right] e^{i k_{z} d} .
\end{gathered}
$$


It is a little tedious, but one can check the unitary condition of the scattering matrix by using the composite transfer matrix [Eq. (C3)].

As $d \rightarrow 0$, the composite transfer matrix [Eq. (C3)] reduces to the transfer matrix of the $\mathrm{N} \delta \mid \mathrm{N}$ structure, and the corresponding reduced scattering matrix reads as follows:

$$
\begin{aligned}
& r=r^{\prime}=\left(2 i k_{z}-\hat{\alpha}_{\text {int }}\right)^{-1}\left(\hat{\alpha}_{\text {int }}\right), \\
& t=t^{\prime}=\left(2 i k_{z}-\hat{\alpha}_{\text {int }}\right)^{-1}\left(2 i k_{z}\right) .
\end{aligned}
$$

These reduced scattering matrices [Eqs. (C4)] are identical to the results of Ref. [22] and those of Refs. [19,52]. As $d \rightarrow 0$, one can find that the inversion symmetry appears again and it makes $r=r^{\prime}$ and $t=t^{\prime}$. The pumped charge current trivially vanishes in this case.

As $\hat{\kappa}_{z} \rightarrow k_{z}$, which implies that the sandwiched ferromagnet becomes nonmagnetic metal, so [Eq. (C3)] also reduces to the tranfer matrix for the $\mathrm{N} \delta \mid \mathrm{N}$ structure again. However, the center of the interfacial spin-flip potential is shifted to $(z=d)$ compared to the previous result, so it results in the scattering matrix with the shifted scatterer $(z=d)$. As a result, the scattering matrix reads as follows:

$$
\begin{gathered}
\hat{r}=e^{-2 i k_{z} d}\left(2 i k_{z}-\hat{\alpha}_{\text {int }}\right)^{-1}\left(\hat{\alpha}_{\text {int }}\right), \\
\hat{r}^{\prime}=e^{2 i k_{z} d}\left(2 i k_{z}-\hat{\alpha}_{\text {int }}\right)^{-1}\left(\hat{\alpha}_{\text {int }}\right), \\
\hat{t}=\hat{t}^{\prime}=\left(2 i k_{z}-\hat{\alpha}_{\text {int }}\right)^{-1}\left(2 i k_{z}\right),
\end{gathered}
$$

where we can see that the transmission matrices $\left(\hat{t}\right.$ and $\left.\hat{t}^{\prime}\right)$ are not changed, but the reflection matrix $\hat{r}\left(\hat{r}^{\prime}\right)$ gains the phase factor $e^{-2 i k_{z} d}\left(e^{2 i k_{z} d}\right)$. It is consistent with the scattering matrix for a translated scatterer [53].

[1] Y. Tserkovnyak, A. Brataas, and G. E. W. Bauer, Enhanced Gilbert Damping in Thin Ferromagnetic Films, Phys. Rev. Lett. 88, 117601 (2002).

[2] Y. Tserkovnyak, A. Brataas, G. E. W. Bauer, and B. I. Halperin, Nonlocal magnetization dynamics in ferromagnetic hybrid nanostructures, Rev. Mod. Phys. 77, 1375 (2005).

[3] A. Brataas, Y. Tserkovnyak, and G. E. W. Bauer, Scattering Theory of Gilbert Damping, Phys. Rev. Lett. 101, 037207 (2008).

[4] A. Brataas, Y. V. Nazarov, and G. E. W. Bauer, Finite-Element Theory Of Transport in Ferromagnet-Normal Metal Systems, Phys. Rev. Lett. 84, 2481 (2000).

[5] A. Brataas, Y. Nazarov, and G. Bauer, Spin-transport in multiterminal normal metal-ferromagnet systems with non-collinear magnetizations, Eur. Phys. J. B 22, 99 (2001).

[6] A. Brataas, G. E. W. Bauer, and P. J. Kelly, Non-collinear magnetoelectronics, Phys. Rep. 427, 157 (2006).

[7] J. C. Slonczewski, Current-driven excitation of magnetic multilayers, J. Magn. Magn. Mater. 159, L1 (1996).

[8] L. Berger, Emission of spin waves by a magnetic multilayer traversed by a current, Phys. Rev. B 54, 9353 (1996).

[9] M. D. Stiles and A. Zangwill, Anatomy of spin-transfer torque, Phys. Rev. B 66, 014407 (2002).

[10] D. C. Ralph and M. D. Stiles, Spin transfer torques, J. Magn. Magn. Mater. 320, 1190 (2008).

[11] I. M. Miron, K. Garello, G. Gaudin, P. J. Zermatten, M. V. Costache, S. Auffret, S. Bandiera, B. Rodmacq, A. Schuhl, and P. Gambardella, Perpendicular switching of a single ferromagnetic layer induced by in-plane current injection, Nature (London) 476, 189 (2011).

[12] L. Liu, C. F. Pai, Y. Li, H. W. Tseng, D. C. Ralph, and R. A. Buhrman, Spin-torque switching with the giant spin Hall effect of tantalum, Science 336, 555 (2012).
[13] J.-C. Rojas-Sánchez, N. Reyren, P. Laczkowski, W. Savero, J.-P. Attané, C. Deranlot, M. Jamet, J.-M. George, L. Vila, and H. Jaffrès, Spin Pumping and Inverse Spin Hall Effect in Platinum: The Essential Role Of Spin-Memory Loss at Metallic Interfaces, Phys. Rev. Lett. 112, 106602 (2014).

[14] V. P. Amin and M. D. Stiles, Spin transport at interfaces with spin-orbit coupling: Formalism, Phys. Rev. B 94, 104419 (2016).

[15] K. D. Belashchenko, A. A. Kovalev, and M. van Schilfgaarde, Theory of Spin Loss at Metallic Interfaces, Phys. Rev. Lett. 117, 207204 (2016).

[16] P. M. Haney and M. D. Stiles, Current-Induced Torques in the Presence of Spin-Orbit Coupling, Phys. Rev. Lett. 105, 126602 (2010).

[17] P. M. Haney, H. W. Lee, K. J. Lee, A. Manchon, and M. D. Stiles, Current induced torques and interfacial spin-orbit coupling: Semiclassical modeling, Phys. Rev. B 87, 174411 (2013).

[18] G. Yu, P. Upadhyaya, Y. Fan, J. G. Alzate, W. Jiang, K. L. Wong, S. Takei, S. A. Bender, L. T. Chang, Y. Jiang, M. Lang, J. Tang, Y. Wang, Y. Tserkovnyak, P. K. Amiri, and K. L. Wang, Switching of perpendicular magnetization by spin-orbit torques in the absence of external magnetic fields, Nat. Nanotechnol. 9, 548 (2014).

[19] V. P. Amin, J. Zemen, and M. D. Stiles, Interface-Generated Spin Currents, Phys. Rev. Lett. 121, 136805 (2018).

[20] G. G. Baez Flores, A. A. Kovalev, M. van Schilfgaarde, and K. D. Belashchenko, Generalized magnetoelectronic circuit theory and spin relaxation at interfaces in magnetic multilayers, Phys. Rev. B 101, 224405 (2020).

[21] K. Chen and S. Zhang, Spin Pumping in the Presence of SpinOrbit Coupling, Phys. Rev. Lett. 114, 126602 (2015).

[22] K.-W. Kim, K.-J. Lee, J. Sinova, H.-W. Lee, and M. D. Stiles, Spin-orbit torques from interfacial spin-orbit 
coupling for various interfaces, Phys. Rev. B 96, 104438 (2017).

[23] S.-H. Chen, C.-R. Chang, J. Q. Xiao, and B. K. Nikolić, Spin and charge pumping in magnetic tunnel junctions with precessing magnetization: A nonequilibrium Green function approach, Phys. Rev. B 79, 054424 (2009).

[24] K. M. Hals, A. Brataas, and Y. Tserkovnyak, Scattering theory of charge-current-induced magnetization dynamics, Europhys. Lett. 90, 47002 (2010).

[25] K. M. D. Hals and A. Brataas, Spin-motive forces and currentinduced torques in ferromagnets, Phys. Rev. B 91, 214401 (2015).

[26] G. Tatara, N. Nakabayashi, and K.-J. Lee, Spin motive force induced by Rashba interaction in the strong $s d$ coupling regime, Phys. Rev. B 87, 054403 (2013).

[27] C. Ciccarelli, K. M. Hals, A. Irvine, V. Novak, Y. Tserkovnyak, H. Kurebayashi, A. Brataas, and A. Ferguson, Magnonic charge pumping via spin-orbit coupling, Nat. Nanotechnol. 10, 50 (2015).

[28] A. Azevedo, R. O. Cunha, F. Estrada, O. Alves Santos, J. B. S. Mendes, L. H. Vilela-Leão, R. L. Rodríguez-Suárez, and S. M. Rezende, Electrical detection of ferromagnetic resonance in single layers of permalloy: Evidence of magnonic charge pumping, Phys. Rev. B 92, 024402 (2015).

[29] F. Mahfouzi, J. Fabian, N. Nagaosa, and B. K. Nikolić, Charge pumping by magnetization dynamics in magnetic and semimagnetic tunnel junctions with interfacial Rashba or bulk extrinsic spin-orbit coupling, Phys. Rev. B 85, 054406 (2012).

[30] T. Nan, S. Emori, C. T. Boone, X. Wang, T. M. Oxholm, J. G. Jones, B. M. Howe, G. J. Brown, and N. X. Sun, Comparison of spin-orbit torques and spin pumping across $\mathrm{NiFe} / \mathrm{Pt}$ and $\mathrm{NiFe} / \mathrm{Cu} / \mathrm{Pt}$ interfaces, Phys. Rev. B 91, 214416 (2015).

[31] H. L. Wang, C. H. Du, Y. Pu, R. Adur, P. C. Hammel, and F. Y. Yang, Scaling of Spin Hall Angle in 3d, 4d, and 5d Metals from $\mathrm{Y}_{3} \mathrm{Fe}_{5} \mathrm{O}_{12}$ /Metal Spin Pumping, Phys. Rev. Lett. 112, 197201 (2014).

[32] Y. Tserkovnyak and H. Ochoa, Generalized boundary conditions for spin transfer, Phys. Rev. B 96, 100402(R) (2017).

[33] K.-W. Kim and K.-J. Lee, Generalized Spin Drift-Diffusion Formalism in the Presence of Spin-Orbit Interaction of Ferromagnets, Phys. Rev. Lett. 125, 207205 (2020).

[34] P. W. Brouwer, Scattering approach to parametric pumping, Phys. Rev. B 58, R10135 (1998).

[35] M. Büttiker, H. Thomas, and A. Prêtre, Current partition in multiprobe conductors in the presence of slowly oscillating external potentials, Z. Phys. B: Condens. Matter 94, 133 (1994).

[36] M. Moskalets and M. Buttiker, Floquet scattering theory of quantum pumps, Phys. Rev. B 66, 205320 (2002).

[37] A. Kiss, L. Szolnoki, and F. Simon, The Elliott-Yafet theory of spin relaxation generalized for large spin-orbit coupling, Sci. Rep. 6, 22706 (2016).

[38] Q. Xie, W. Lin, B. Yang, X. Shu, S. Chen, L. Liu, X. Yu, M. B. Breese, T. Zhou, M. Yang, Z. Zhang, S. Wang, H. Yang, J. Chai, $\mathrm{X}$. Han, and J. Chen, Giant enhancements of perpendicular magnetic anisotropy and spin-orbit torque by a $\mathrm{MoS}_{2}$ layer, Adv. Mater. 31, 1900776 (2019).
[39] Y.-C. Lau, Z. Chi, T. Taniguchi, M. Kawaguchi, G. Shibata, N. Kawamura, M. Suzuki, S. Fukami, A. Fujimori, H. Ohno, and M. Hayashi, Giant perpendicular magnetic anisotropy in Ir/Co/Pt multilayers, Phy. Rev. Mater. 3, 104419 (2019).

[40] J.-X. Yu and J. Zang, Giant perpendicular magnetic anisotropy in Fe/III-V nitride thin films, Sci. Adv. 4, eaar7814 (2018).

[41] G. Wu, D. Wu, Y. Ren, Q. Y. Jin, and Z. Zhang, Topological surface state manipulation of magnetic damping and surface anisotropy in topological insulator/nonmagnet/CoFe heterostructures, Phys. Rev. B 103, 014419 (2021).

[42] K. Chen and S. Zhang, Spin pumping induced electric voltage, IEEE Magn. Lett. 6, 3000304 (2015).

[43] L. Bai, P. Hyde, Y. S. Gui, C. M. Hu, V. Vlaminck, J. E. Pearson, S. D. Bader, and A. Hoffmann, Universal Method for Separating Spin Pumping from Spin Rectification Voltage of Ferromagnetic Resonance, Phys. Rev. Lett. 111, 217602 (2013).

[44] M. V. Costache, M. Sladkov, S. M. Watts, C. H. van der Wal, and B. J. van Wees, Electrical Detection of Spin Pumping Due to the Precessing Magnetization of a Single Ferromagnet, Phys. Rev. Lett. 97, 216603 (2006).

[45] X. Wang, G. E. W. Bauer, B. J. van Wees, A. Brataas, and Y Tserkovnyak, Voltage Generation by Ferromagnetic Resonance at a Nonmagnet to Ferromagnet Contact, Phys. Rev. Lett. 97, 216602 (2006).

[46] O. Mosendz, J. E. Pearson, F. Y. Fradin, G. E. W. Bauer, S. D. Bader, and A. Hoffmann, Quantifying Spin Hall Angles from Spin Pumping: Experiments and Theory, Phys. Rev. Lett. 104, 046601 (2010).

[47] M. Harder, Y. Gui, and C. M. Hu, Electrical detection of magnetization dynamics via spin rectification effects, Phys. Rep. 661, 1 (2016).

[48] T. Moriyama, R. Cao, X. Fan, G. Xuan, B. K. Nikolić, Y. Tserkovnyak, J. Kolodzey, and J. Q. Xiao, Tunnel Barrier Enhanced Voltage Signal Generated by Magnetization Precession of a Single Ferromagnetic Layer, Phys. Rev. Lett. 100, 067602 (2008).

[49] M. Evelt, H. Ochoa, O. Dzyapko, V. E. Demidov, A. Yurgens, J. Sun, Y. Tserkovnyak, V. Bessonov, A. B. Rinkevich, and S. O. Demokritov, Chiral charge pumping in graphene deposited on a magnetic insulator, Phys. Rev. B 95, 024408 (2017).

[50] A. Suresh, U. Bajpai, and B. K. Nikolić, Magnon-driven chiral charge and spin pumping and electron-magnon scattering from time-dependent quantum transport combined with classical atomistic spin dynamics, Phys. Rev. B 101, 214412 (2020).

[51] F. Mahfouzi, B. K. Nikolić, S. H. Chen, and C. R. Chang, Microwave-driven ferromagnet-topological-insulator heterostructures: The prospect for giant spin battery effect and quantized charge pump devices, Phys. Rev. B 82, 195440 (2010).

[52] V. P. Amin, P. M. Haney, and M. D. Stiles, Interfacial spin-orbit torques, J. Appl. Phys. 128, 151101 (2020).

[53] J. E. Avron, A. Elgart, G. M. Graf, and L. Sadun, Geometry, statistics, and asymptotics of quantum pumps, Phys. Rev. B 62, R10618 (2000). 